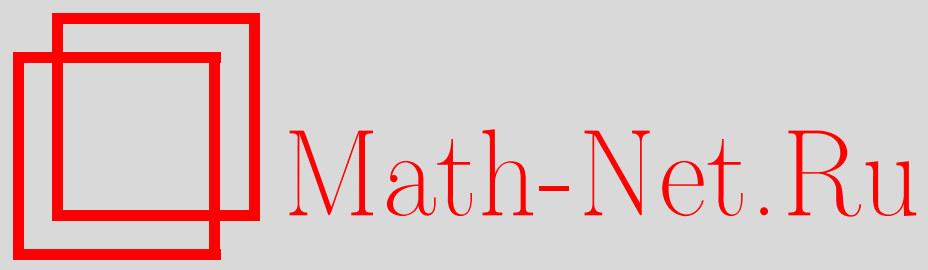

Д. А. Панов, Многокомпонентные псевдопериодические отображения, Функи. анализ и его прил., 1996, том 30, выпуск 1, 30-38

DOI: https://doi.org/10.4213/faa479

Использование Общероссийского математического портала MathNet.Ru подразумевает, что вы прочитали и согласны с пользовательским соглашением

http://www . mathnet.ru/rus/agreement

Параметры загрузки:

IP : 44.207 .124 .84

26 апреля 2023 г., 15:07:11

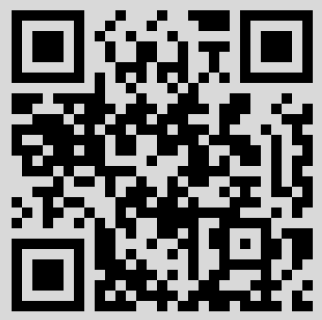


Функииональный анализ и его приложения

1996, т. 30, вып. 1 , с. $30-38$

УДК 517.9

\title{
Многокомпонентные псевдопериодические отображения
}

\author{
(C) 1996. Д. А. ПАнов
}

\section{Введение}

Отображение $P$ из $\mathbb{R}^{n}$ в $\mathbb{R}^{m}$ называется периодическим, если $P(x+t)=P(x)$ для любого $x \in \mathbb{R}^{n}$ и любого $t \in \mathbb{Z}^{n}$.

ОПРЕДЕЛЕНИЕ 1 . Отображение из $\mathbb{R}^{n}$ в $\mathbb{R}^{m}$, представимое в виде суммы линейного и периодического отображений, называется псевдопериодическим.

Мы ограничимся рассмотрением псевдопериодических отображений из $\mathbb{R}^{n}$ в $\mathbb{R}^{n-1}$, под действием которых целочисленная решетка $\mathbb{Z}^{n}$ переходит во всюду плотное подмножество в $\mathbb{R}^{n-1}$.

Регулярной линией уровня отображения из $\mathbb{R}^{n}$ в $\mathbb{R}^{n-1}$ называется прообраз регулярного значения этого отображения. Каждая такая линия распадается на семейство неограниченных компонент, диффеоморфных прямой, и семейство ограниченных компонент, диффеоморфных окружности. В [3] было доказано, что все регулярные линии уровня псевдопериодического отображения содержат одно и то же число неограниченных компонент (и это число нечетно), но не были известны примеры отображений, регулярные линии уровня которых содержат больше одной неограниченной компоненты. В $\S 1$ такой пример построен.

Теорема 1. Существуют псевдопериодические отображения из $\mathbb{R}^{3}$ в $\mathbb{R}^{2}$, регулярные линии уровня которых содержат сколь угодно большое число неограниченньх компонент.

В связи с этим введем определение.

ОПредЕлЕниЕ 2. Псевдопериодическое отображение из $\mathbb{R}^{n}$ в $\mathbb{R}^{n-1}$ называется $k$-компонентнылм, если все регулярные линии уровня этого отображения содержат по $k$ неограниченных компонент.

В отличие от больших $n(n>2)$ при $n=2$ все псевдопериодические отображения однокомпонентны. Этот факт был доказан в [1] и имеет многомерное обобщение, которое было высказано в [2] в качестве гипотезы: если $F=$ $\left(f_{1}, \ldots, f_{n-1}\right)$ - псевдопериодическое отображение из $\mathbb{R}^{n}$ в $\mathbb{R}^{n-1}$ и функции $f_{2}, \ldots, f_{n-1}$ линейны, то $F-$ однокомпонентное отображение. Здесь доказывается более общее утверждение.

Tеорема 2. Пусть $F=\left(f_{1}, \ldots, f_{n-1}\right)-$ nсевдопериодическое отображение из $\mathbb{R}^{n}$ в $\mathbb{R}^{n-1}$. Пусть для некоторых $a_{2}, \ldots, a_{n-1}$ поверхность

$$
M=f_{2}^{-1}\left(a_{2}\right) \cap \cdots \cap f_{n-1}^{-1}\left(a_{n-1}\right)
$$


регулярна и является объединением компоненты $\pi$, диффеоморфной плоскости, и семейства ограниченных поверхностей. Тогда $F$ - однокомпонентное отображение.

ОПРЕДЕЛЕНИЕ 3. $k$-компонентное отображение называется устойчивъим, если при малых возмущениях оно остается $k$-компонентным.

Все однокомпонентные отображения являются устойчивыми. Этот факт вытекает из следующего утверждения.

ТЕОремА 3. Количество неограниченных компонент регулярной линии уровня псевдопериодического отображения не может увеличиться при мальх возмущениях отображения.

Из этой теоремы также следует, что существуют неустойчивые многокомпонентные отображения, при сколь угодно малых возмущениях которых число неограниченных компонент регулярной линии уровня отображения может уменьшаться. Устойчивость и неустойчивость отображений, по-видимому, связаны со следующим фактом.

ТеОремА 4. Пусть $F$ есть $k$-компонентное псевдопериодическое отображение. Рассмотрим множество $\bar{N}$, являюшееся замыканием объединения всех неограниченных компонент регулярных линий уровня отображения $F$. Число компонент связности множества $\bar{N}$ не превосходит $k$.

Возможно, $k$-компонентное отображение устойчиво в том и только в том случае, когда соответствующее множество $\bar{N}$ имеет ровно $k$ компонент связности.

Выражаю признательность В. И. Арнольду, И. А. Дынникову и Ю. В. Чеканову за постановку задачи и полезные обсуждения.

\section{§1. Пример многокомпонентного отображения}

В этом параграфе будет построен пример многокомпонентного отображения из $\mathbb{R}^{3}$ в $\mathbb{R}^{2}$. Для этого нам потребуется несколько вспомогательных конструкций и утверждений.

Определим сначала некоторое расслоение пространства $\mathbb{R}^{3}$. Через $V_{0}$ обозначим одномерный остов целочисленной решетки в $\mathbb{R}^{3}$ - множество, являющееся объединением прямых, параллельных координатным осям и проходящих через целочисленные точки. Через $V_{1}$ обозначим сдвиг $V_{0}$ на вектор $(1 / 2,1 / 2,1 / 2)$. В пространстве $\mathbb{R}^{3} \backslash\left(V_{0} \cup V_{1}\right)$ введем структуру расслоения. Базой этого расслоения будет интервал $(0,1)$, а слоем $\widetilde{V}(a)$, $a \in(0,1)$, - множество точек, удаленных от остова $V_{0}$ на расстояние $a / 2$ в метрике $\rho(x, y)=\max _{i}\left|x_{i}-y_{i}\right|$.

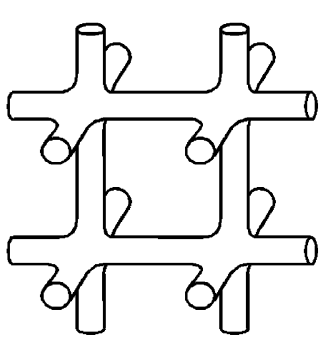

Рис. 1

Ясно, что все слои гомеоморфны и расслоение тривиально. Конечно, слои $\widetilde{V}(a)$ не являются гладкими поверхностями, но малой периодической деформацией их можно сделать гладкими. Для этих гладких слоев введем новое обозначение $V(a)$. Для малого $\varepsilon$ типичный слой $V(\varepsilon)$ изображен на рис. 1. По непрерывности это расслоение можно продолжить до отображения $\mathbb{R}^{3} \rightarrow[0,1]$, положив $V(0)=V_{0}$, $V(1)=V_{1}$. Введем еще обозначение $V[a, b]=\bigcup_{t \in[a, b]} V(t)$. 
ПреДлОЖЕНИЕ 1 . Пусть $L=\left(l_{1}, l_{2}\right)$ - линейное отображение из $\mathbb{R}^{3}$ в $\mathbb{R}^{2}$. Тогда существуют число $\varepsilon>0$ и псевдопериодическое отображение $F=\left(f_{1}, f_{2}\right)$ с линейной частью $L$, такие, что каждая регулярная линия уровня отображения $F$ содержит неограниченную компоненту, лежащую вне $V[0, \varepsilon]$.

ДокАЗАТЕльство. Ограничимся случаем, когда плоскость $l_{1}^{-1}(0)$ образует ненулевые углы с координатными осями. Выберем $\varepsilon$ настолько малым, что диаметры связных кусков пересечения множества $V[0,2 \varepsilon]$ с каждой плоскостью $l_{1}^{-1}(c), c \in \mathbb{R}$, меньше 1 .

Положим $f_{1}=l_{1}$. Из выбора $\varepsilon$ следует, что если функция $f_{1}$ постоянна на связной кривой $\gamma$ и $\gamma \subset V[0,2 \varepsilon]$, то диаметр кривой $\gamma$ не больше 1 . Положим

$$
s=2\left(\max _{|x-y| \leqslant 1}\left|l_{2}(x)-l_{2}(y)\right|\right) .
$$

Возьмем периодическую функцию $P$, равную $s$ на поверхности $V(\varepsilon)$ и 0 на поверхности $V(2 \varepsilon)$. Положим $f_{2}=l_{2}+P$.

Рассмотрим теперь неограниченную компоненту $\gamma=\gamma(t)$ произвольной линии уровня отображения $F=\left(f_{1}, f_{2}\right)$. Допустим, что в некоторый момент $t_{1}$ кривая $\gamma$ пересекает поверхность $V(\varepsilon)$. Кривая $\gamma$ не может целиком содержаться в $V[0,2 \varepsilon]$, так как любой ее связный кусок, лежащий в $V[0,2 \varepsilon]$, должен иметь диаметр меньше 1 . Поэтому существует такой момент $t_{0}, t_{0}<t_{1}$, когда $\gamma$ пересекает поверхность $V(2 \varepsilon)$, и расстояние между точками $\gamma\left(t_{0}\right)$ и $\gamma\left(t_{1}\right)$ меньше 1 . Но тогда, с одной стороны, $f_{2}\left(\gamma\left(t_{0}\right)\right)=f_{2}\left(\gamma\left(t_{1}\right)\right)$, а с другой стороны, в силу выбора (1) константы $s$ имеем $f_{2}\left(\gamma\left(t_{0}\right)\right)<f_{2}\left(\gamma\left(t_{1}\right)\right)$. Полученное противоречие доказывает, что $\gamma$ не может пересекать $V(\varepsilon)$. Отсюда следует, что $\gamma$ лежит целиком вне $V[0, \varepsilon]$. Для завершения доказательства остается воспользоваться результатом из [2] о том, что любая регулярная линия уровня псевдопериодического отображения содержит неограниченную компоненту. Предложение доказано.

Можно усилить предложение и более точно локализовать неограниченные компоненты, а именно:

СлЕДСТВИЕ 1. Пусть задано линейное отображение $L=\left(l_{1}, l_{2}\right)$ и положительное число $\delta$. Тогда существуют периодические функиии $p_{1}$ u $p_{2} c$ носителями, лежащими в $V[1-2 \delta, 1]$, такие, ито каждая регулярная линия уровня отображения $G=\left(l_{1}+p_{1}, l_{2}+p_{2}\right)$ содержит неограниченную компоненту, лежашую в $V[1-\delta, 1]$.

ДокАЗАТЕЛЬСтво. Для линейного отображения $L$ найдем псевдопериодическое отображение $F=\left(f_{1}, f_{2}\right)$ и число $\varepsilon$, удовлетворяющие заключению предложения 1.

Через $\varphi$ обозначим диффеоморфизм отрезка $[0,1]$ в себя, такой, что $\varphi(\varepsilon)=$ $1-\delta$ и $\varphi(x)=x$ при $x \in[0, \varepsilon / 2] \cup[1-\delta / 2,1]$. Диффеоморфизм $\varphi$ индуцирует послойный диффеоморфизм $\Phi: \mathbb{R}^{3} \rightarrow \mathbb{R}^{3}$

$$
\Phi(V(a))=V(\varphi(a)) .
$$

При этом $\mathbb{R}^{3} \backslash V[0, \varepsilon]$ переходит внутрь $V[1-\delta, 1]$. Через $h$ обозначим функцию на $[0,1]$, такую, что $h(x)=0$ при $x \in[0,1-2 \delta]$ и $h(x)=1$ при $x \in[1-\delta, 1]$, и через $H$ - ее послойное поднятие в $\mathbb{R}^{3}, H(V(a))=h(a)$. 
Теперь, используя предложение 1, получаем, что любая регулярная линия уровня отображения $G^{\prime}=\left(f_{1} \circ \Phi^{-1}, f_{2} \circ \Phi^{-1}\right)=F \circ \Phi^{-1}$ имеет неограниченную компоненту, лежащую в $V[1-\delta, 1]$. Так как диффеоморфизм $\Phi$ периодический, он переводит любую псевдопериодическую функцию в псевдопериодическую функцию с той же линейной частью. Значит,

$$
f_{1} \circ \Phi^{-1}=l_{1}+p_{1}^{\prime}, \quad f_{2} \circ \Phi^{-1}=l_{2}+p_{2}^{\prime},
$$

где $p_{1}^{\prime}$ и $p_{2}^{\prime}$ - периодические функции. Остается положить $p_{1}=H \cdot p_{1}^{\prime}$ и $p_{2}=$ $H \cdot p_{2}^{\prime}$ и заметить, что $p_{1}$ и $p_{1}^{\prime}, p_{2}$ и $p_{2}^{\prime}$ совпадают на $V[1-\delta, 1]$. Искомые функции $p_{1}$ и $p_{2}$ построены, следствие доказано.

Опуская детали, полученный результат можно сформулировать короче: любое псевдопериодическое отображение можно изменить вблизи одномерного остова $V_{0}=V(0)$ таким образом, чтобы в состав любой регулярной линии уровня полученного отображения входила неограниченная компонента, расположенная вблизи $V_{0}$.

Перейдем теперь непосредственно к построению примера многокомпонентного псевдопериодического отображения. Разнесем остов $V_{0}$ при помощи векторов $m_{i}=(i / k, i / k, i / k), 0 \leqslant i<k$ по $\mathbb{R}^{3}$, положив на этот раз $V_{i}=V_{0}+m_{i}$. Возьмем любое псевдопериодическое отображение из $\mathbb{R}^{3}$ в $\mathbb{R}^{2}$. Используя следствие 1 , изменим это отображение вблизи каждого остова $V_{i}$ так, чтобы любая регулярная линия уровня полученного отображения содержала неограниченную компоненту, расположенную вблизи $V_{i}$. Число таких компонент будет совпадать с числом остовов $V_{i}$ и равно, таким образом, $k$. Пример построен.

ЗАмЕчАНИЕ. Построенное псевдопериодическое отображение является устойчивым в том смысле, что линии уровня слабо возмущенного отображения также будут содержать не менее $k$ неограниченных компонент.

Все результаты этого параграфа допускают обобщение на многомерный случай.

ТЕОРема $1^{\prime}$. При $n \geqslant 3 u m \geqslant 2, m<n$, существует псевдопериодическое отображение $F=\left(f_{1}, \ldots, f_{m}\right)$ из $\mathbb{R}^{n}$ в $\mathbb{R}^{m}$, регулярные поверхности уровня которого содержат сколь угодно большое число неограниченньх компонент. При этом $m-2$ функций $f_{3}, \ldots, f_{m}$ могут быть взяты линейныли.

\section{§2. Изменение числа компонент при малых возмущениях}

В этом параграфе будет доказана теорема 3, сформулированная во введении. Для доказательства нам потребуются две леммы, вытекающие из результатов [3].

Лемма 1. Пусть $F$ - псевдопериодическое отображение из $\mathbb{R}^{n}$ в $\mathbb{R}^{n-1}$ c линейной частью $L, a_{0}$ - регулярное значение отображения $F u J-$ неограниченная компонента линии $F^{-1}\left(a_{0}\right)$. Тогда ортогональная проекиия кривой $J$ на прямую $L^{-1}(0)$ покрывает всю прямую $L^{-1}(0)$.

Лемма 2. Пусть $F$ - псевдопериодическое отображение из $\mathbb{R}^{n}$ в $\mathbb{R}^{n-1} u$ $F^{-1}(a)$ - регулярная линия уровня этого отображения. Тогда существует 
гиперповерхность П, трансверсальная $F^{-1}(a)$, пересекающая каждую неограниченную компоненту $F^{-1}(a)$ ровно в одной точке и не пересекаюшая ни одной из ограниченных компонент $F^{-1}(a)$. При этом П пересекает любую кривую, ортогональная проекчия которой на прямую $L^{-1}(0)$ покрывает всю прямую $L^{-1}(0)$.

Приступим теперь к доказательству теоремы 3.

ДокАЗАТЕЛЬСтво. Пусть $F$ есть $k$-компонентное отображение из $\mathbb{R}^{n}$ в $\mathbb{R}^{n-1}, a_{0}$ - его регулярное значение и $J^{1}, \ldots, J^{k}$ - неограниченные компоненты линии $F^{-1}\left(a_{0}\right)$. По отображению $F$ и линии $F^{-1}\left(a_{0}\right)$ построим гиперповерхность П, удовлетворяющую заключению леммы 2. Обозначим точку пересечения гиперповерхности $\Pi$ с компонентой $J^{i}$ через $x_{i}$. Пусть $F_{\Pi}-$ ограничение отображения $F$ на гиперповерхность П. Из регулярности линии $F^{-1}\left(a_{0}\right)$ и из того, что она трансверсальна $\Pi$, следует, что отображение $F_{\Pi}$ будет локальным диффеоморфизмом в точках $x_{i}$. Пусть $G$ есть $C^{1}$-малая периодическая деформация отображения $F$. Понятно, что отображение $G_{\Pi}$ будет $C^{1}$-малой деформацией отображения $F_{\Pi}$. Из того что $a_{0}$ является регулярным значением для $F_{\Pi}$, следует, что оно будет регулярным и для $G_{\Pi}$. Кроме того, $\# F_{\Pi}^{-1}\left(a_{0}\right)=\# G_{\Pi}^{-1}\left(a_{0}\right)=k$. Очевидно, что при $a$, близких к $a_{0}$, множество $G_{\Pi}^{-1}(a)$ также будет состоять из $k$ точек.

Итак, линия $G^{-1}(a)$ пересекается с $\Pi$ в $k$ точках при значениях $a$, близких к $a_{0}$. Пусть $a_{1}$ - регулярное значение отображения $G$, близкое к $a_{0}$. Из объединения лемм 1 и 2 следует, что каждая неограниченная компонента линии $G^{-1}\left(a_{1}\right)$ пересекает П. Поэтому у линии $G^{-1}\left(a_{1}\right)$ не больше $k$ неограниченных компонент. Теорема доказана.

СЛЕдСТВИЕ 2. Однокомпонентные псевдопериодические отображения являются устойчивьлми.

\section{§3. Доказательство теоремы 2}

Пусть $F$ есть $k$-компонентное псевдопериодическое отображение из $\mathbb{R}^{n}$ в $\mathbb{R}^{n-1}$ с линейной частью $L$. Неограниченные компоненты регулярной линии уровня $F^{-1}(a)$ обозначим через $J_{a}^{1}, \ldots, J_{a}^{k}$.

ОПРЕДЕлЕНИЕ 4. Кривая $\gamma$ называется $d$-деформаиией прямой $l$, если кривую $\gamma$ и прямую $l$ можно так параметризовать, что $|\gamma(t)-l(t)|<d$ для любого $t$.

В работе [3] был доказан следующий результат.

Лемма 3. Пусть $F$ есть $k$-компонентное отображение из $\mathbb{R}^{n}$ в $\mathbb{R}^{n-1} c$ линейной частью $L$. Тогда существует постоянная $d>0$, такая, что для любого регулярного значения а каждая линия $J_{a}^{i}$ является $d$-деформачией прямой $L^{-1}(a)$.

Аналог этого утверждения для ограниченных компонент выглядит следующим образом.

Лемма 4. Существует постоянная $d>0$, такая, что диаметр ограниченных компонент любой регулярной линии $F^{-1}(a)$ меньше $d$.

Доказательство этой леммы аналогично доказательству леммы 3 и легко извлекается из [3]. 
Каждая из этих лемм имеет свое следствие.

СЛЕДСТВИЕ 3. Пусть $J$ - неограниченная компонента регулярной линии $F^{-1}(a)$, точка $x$ лежит на J, а точка у не лежит. Тогда существуют окрестности $U(x)$ и $U(y)$ точек $x$ и $y$, такие, что ни одна из неограниченных компонент регулярных линий уровня не пересекает одновременно обе окрестности $U(x)$ и $U(y)$.

СЛЕДСТВИЕ 4. Для яюбой точки $x$, лежащей на неограниченной компоненте J регулярной линии $F^{-1}(a)$, существует ее окрестность $U(x)$, которую не пересекают ограниченные компоненты регулярных линий уровня.

Доказательства этих следствий похожи, поэтому докажем только второе.

ДокАЗАтельство. Пусть $x_{1}, x_{2} \in J$, точка $x$ лежит на $J$ между $x_{1}$ и $x_{2}$ и $\left|x_{1}-x_{2}\right|>2 d$, где $d$ - константа из леммы 4. Рассмотрим трубчатую окрестность $U(x)$ отрезка линии $J$, лежащего между $x_{1}$ и $x_{2}$. Так как $a-$ регулярное значение отображения $F$, можно потребовать, чтобы $U(x)$ расслаивалась линиями уровня отображения $F$ на отрезки, причем диаметр каждого из этих отрезков был не меньше $2 d$. Очевидно, что любая компонента, пересекающая $U(x)$, имеет диаметр, больший $2 d$, и, значит, не может быть ограниченной и регулярной. Следствие доказано.

ОПРЕДЕЛЕНИЕ 5. Открытое подмножество $U$ пространства $\mathbb{R}^{n}$ назовем представительным, если для каждой неограниченной компоненты любой регулярной линии $F^{-1}(a)$ найдется бесконечный набор целочисленных векторов $m_{i}$, таких, что эта компонента пересекается с каждым из множеств $U+m_{i}$.

ПРЕДЛОЖЕНИЕ 2. Пусть $а$ - регулярное значение отображения $F u$ открытое подмножество $U$ в $\mathbb{R}^{n}$ пересекается со всеми неограниченньлми компонентами линии уровня $F^{-1}(a)$. Тогда $U$ является представительHblм.

ДокАЗАТЕЛЬСтво. Пусть $F$ есть $k$-компонентное отображение. В множестве $U$ выделим $k$ открытых подмножеств $u_{1}, \ldots, u_{k}$, каждое из которых пересекается ровно с одной из неограниченных компонент линии $F^{-1}(a)$. Пользуясь следствиями 3 и 4 , множества $u_{1}, \ldots, u_{k}$ можно выбрать так, чтобы ни одна из регулярных неограниченных компонент отображения $F$ не пересекала одновременно двух множеств и никакая из ограниченных не пересекала ни одного из этих множеств. Из выбора $u_{i}$ следует, что если некоторая регулярная линия уровня пересекается с каждым из $u_{i}$, то любая из неограниченных компонент этой линии пересекает одно и только одно $u_{i}$.

Докажем, что множество $\bigcup_{i} u_{i}$ представительное. Пусть $b-$ регулярное значение отображения $F$. Образ решетки $\mathbb{Z}^{n}$ при отображении $F$ всюду плотен; поэтому существует бесконечное число целочисленных векторов $m$, таких, что точка $b-F(m)$ принадлежит множеству $\bigcap_{i} F\left(u_{i}\right)$. Регулярная линия уровня $F^{-1}(b-F(m))$ будет пересекать каждое из множеств $u_{i}$; поэтому все неограниченные компоненты этой линии будут пересекаться с множеством $\bigcup_{i} u_{i}$. Линия уровня $F^{-1}(b)$ получается из линии $F^{-1}(b-F(m))$ сдвигом на вектор $m$. Поэтому для каждого $m$ все неограниченные компоненты линии $F^{-1}(b)$ будут 
пересекаться с множеством $\bigcup_{i} u_{i}+m$. Это показывает, что множество $\bigcup_{i} u_{i}$ представительное, а значит, и содержащее его множество $U$ тоже представительное. Предложение доказано.

Теперь все готово для доказательства теоремы 2.

ДокАЗАтЕльство. Пусть $a_{1}$ - некритическое значение ограничения $f_{1}$ на $M$. Тогда $a=\left(a_{1}, \ldots, a_{n-1}\right)$ - регулярное значение отображения $F$ вследствие регулярности поверхности $M$. Выберем на каждой из неограниченных компонент $J_{a}^{i}$ линии $F^{-1}(a)$ по точке $x_{i}$. Используя следствие 4 , для каждого $i$ выберем такой диск $D_{i}$ с центром в $x_{i}$, чтобы все компоненты регулярных линий уровня, пересекающие этот диск, были неограниченными. Через $D_{i / 2}$ обозначим диск вдвое меньшего диаметра с центром в точке $x_{i}$. Из предложения 2 следует, что объединение дисков $D_{i / 2}$ будет представительным подмножеством.

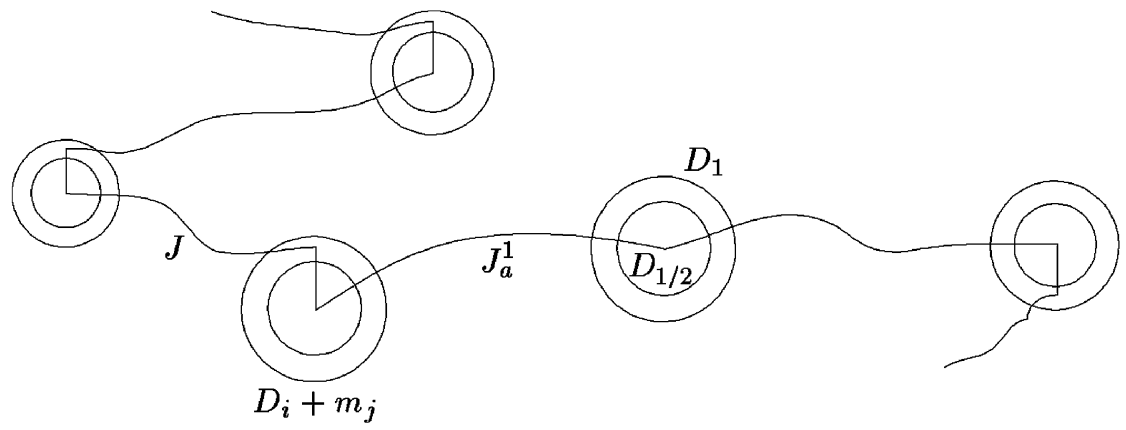

Рис. 2

Построим следующее отображение $\varphi: \mathbb{R} \rightarrow \pi$. Положим $f(0)=x_{1}$. С возрастанием $t$ точка $\varphi(t)$ движется вдоль $J_{a}^{1}$ до пересечения с одним из дисков вида $D_{i / 2}+m_{j}$ (рис. 2). После этого происходит переход из этого диска в больший диск $D_{i}+m_{j}$ таким образом, что при этом переходе точка $\varphi(t)$ попадает на новую неограниченную компоненту $J$ некоторой регулярной линии уровня, и значение $f_{1}(\varphi(t))$ возрастает. Этот переход мы назовем пересадкой. После пересадки точка $\varphi(t)$ движется вдоль $J$ до попадания в следующий диск, и далее процесс повторяется циклически. В силу псевдопериодичности отображения $F$ и условия регулярности можно выбрать начальные диски $D_{i}$ и пересадки таким образом, чтобы возрастание $f_{1}$ при каждой пересадке превосходило некоторую постоянную $\varepsilon>0$. При отрицательных $t$ определим $\varphi(t)$ симметричным образом (рис. 2). Ясно, что функция $f_{1}(\varphi(t))$ монотонна и $f_{1}(\varphi(t)) \rightarrow \pm \infty$ при $t \rightarrow \pm \infty$.

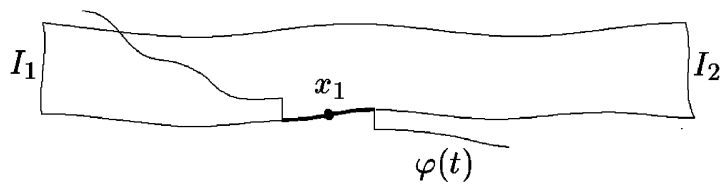

Рис. 3

Покажем, что построенная кривая $\varphi(t)$ пересекает любую неограниченную регулярную компоненту $J_{b}$, лежащую на $\pi$. Для этого нам потребуется следующий простой факт, который мы приводим без доказательства. Пусть $J_{b}, J_{c} \subset \pi$ 
- две неограниченные регулярные компоненты. Тогда существует постоянная $D>0$, такая, что из любой точки одной компоненты можно перейти на другую компоненту по пути длины, меньшей $D$, лежащему в $\pi$. Конечно, $\varphi(t)$ пересекает $J_{a}^{1}$ : по построению $\varphi(0)=x_{1} \in J_{a}^{1}$. Пусть $J_{b}-$ другая регулярная компонента, $J_{b} \subset \pi$. Рассмотрим четырехугольник, составленный из отрезков компонент $J_{a}^{1}$ и $J_{b}$ и соединяющих их конщы отрезков $I_{1}$ и $I_{2}$ длины меньше $D$ (рис. 3). Будем считать, что отрезки $I_{1}$ и $I_{2}$ лежат по разные стороны от точки $x_{1}$ и достаточно удалены от нее. По построению кривая $\varphi(t)$ в одном из направлений входит внутрь четырехугольника. Отрезки $I_{1}$ и $I_{2}$ имеют длину, меньшую $D$; поэтому $f_{1}$ ограничена на $I_{1}$ и $I_{2}$ некоторой постоянной $c=c\left(J_{a}^{1}, J_{b}\right)$,

$$
c\left(J_{a}^{1}, J_{b}\right)=\left|a_{1}\right|+\max _{|x-y| \leqslant D}\left|f_{1}(x)-f_{1}(y)\right| .
$$

Пусть $I_{1}$ и $I_{2}$ настолько удалены, что они не пересекаются с $\varphi\left(\left[t_{1}, t_{2}\right]\right)$, где $f_{1}\left(\varphi\left(t_{1}\right)\right)=-c, f_{1}\left(\varphi\left(t_{2}\right)\right)=c$. Тогда из-за монотонности и неограниченности $f_{1}(\varphi(t))$ кривая $\varphi(t)$ не может выйти из четырехугольника через стороны $I_{1}$ и $I_{2}$, а только через сторону $J_{b}$. Итак, $\varphi(t)$ пересекает любую регулярную неограниченную компоненту $J_{b}$, лежащую на поверхности $\pi$.

Для завершения доказательства теоремы заметим, что все неограниченные компоненты $F^{-1}(a)$ лежат на поверхности $M$ и, значит, на ее единственной неограниченной компоненте $\pi$. Функция $f_{1}(\varphi(t))$ монотонна; поэтому $f_{1}(\varphi(t))$ может быть равна $a_{1}$ только при пересечении кривой $\varphi(t)$ с единственной компонентой, а именно с $J_{a}^{1}$. Таким образом, $F^{-1}(a)$ имеет единственную регулярную компоненту, а потому и любая другая регулярная линия уровня имеет ровно одну неограниченную компоненту. Теорема доказана.

\section{§4. Строение множества неограниченных компонент в целом}

Пусть $F$ есть $k$-компонентное отображение из $\mathbb{R}^{n}$ в $\mathbb{R}^{n-1}$. Под действием целочисленных сдвигов линии уровня отображения $F$ переходят в линии уровня и их неограниченные компоненты — в соответствующие неограниченные компоненты. Таким образом, группа $\mathbb{Z}^{n}$ действует и на множестве $N$, являющемся объединением регулярных неограниченных компонент, и на его замыкании $\bar{N}$. При целочисленных сдвигах компоненты связности множества $\bar{N}$ должны переходить в компоненты связности: значит, $\mathbb{Z}^{n}$ действует и на множестве компонент связности множества $\bar{N}$. Приступим теперь к доказательству теоремы 4 , т. е. покажем, что число компонент множества $N$ не превосходит $k$.

ДокАЗАтЕльство. Пусть $a$ - регулярное значение отображения $F$ и $J_{a}^{1}, \ldots, J_{a}^{k}$ - неограниченные компоненты линии $F^{-1}(a)$. Обозначим через $N_{i}$ компоненту связности множества $N$, содержащую линию $J_{a}^{i}$.

Сначала докажем, что при любом целочисленном сдвиге множество $\bigcup_{i} N_{i}$ переходит в себя. Для этого для каждого $i$ выделим открытый диск $D_{i}$, такой, что, во-первых, $D_{i} \cap J_{a}^{i} \neq \varnothing$, во-вторых, отображение $F$ регулярно на всем диске $D_{i}$ и, в-третьих, компоненты регулярных линий уровня, с которыми пересекается диск $D_{i}$, являются неограниченными и ни одна из компонент не пересекает два диска (следствия 3,4$)$. Ясно, что $D_{i} \subset N_{i}$ для любого $i$. 
Выделим в $\mathbb{Z}^{n}$ все такие векторы $m$, что $a+F(m) \subset \bigcap_{i} F\left(D_{i}\right)$. Из того что множество $a+F\left(\mathbb{Z}^{n}\right)$ всюду плотно в $\mathbb{R}^{n-1}$, легко вывести, что выделенные векторы $m$ порождают все $\mathbb{Z}^{n}$. Регулярная линия уровня $F^{-1}(a+F(m))$ пересекается со всеми дисками $D_{i}$; поэтому каждая из ее неограниченных компонент $J_{a}^{i}$ пересекается с некоторым диском $D_{j}$, а значит, с соответствующим множеством $N_{j}$. Значит, множества $N_{i}+m$ и $N_{j}$ пересекаются, а раз пересекаются, то и совпадают. Отсюда следует, что $\bigcup_{i} N_{i}+m=\bigcup_{i} N_{i}$. Так как выделенные векторы $m$ порождают $\mathbb{Z}^{n}$, то и $\bigcup_{i} N_{i}+\mathbb{Z}^{n}=\bigcup_{i} N_{i}$.

Итак, множество $\bigcup_{i} N_{i}$ инвариантно относительно целочисленных сдвигов. Поэтому множество $\bigcup_{i} N_{i}$ содержит все неограниченные компоненты всех линий уровня $F^{-1}\left(a+F\left(\mathbb{Z}^{n}\right)\right)$. Из плотности $F\left(\mathbb{Z}^{n}\right)$ в $\mathbb{R}^{n-1}$ следует, что $\bigcup_{i} N_{i}$ содержит все неограниченные компоненты всех регулярных линий уровня. Отсюда $N \subset \bigcup_{i} N_{i}$ и, следовательно, $\bar{N}=\bigcup_{i} N_{i}$. Теорема доказана.

Отметим, что в теореме 4 не утверждается, что число компонент связности множества $\bar{N}$ равно в точности $k$. По-видимому, этот факт вообще неверен.

ГиПотеЗА. Пусть F есть $k$-компонентное отображение. Соответствующее множество $\bar{N}$ содержит $k$ компонент в том и только в том случае, когда отображение $F$ является устойчивым.

Отметим также следствие, легко вытекающее из теоремы 4: образ каждой из компонент связности множества $\bar{N}$ под действием отображения $F$ покрывает все $\mathbb{R}^{n-1}$.

\section{ЛиТЕРАТУРА}

1. Арнольд В. И. Топологические и эргодические свойства замкнутых 1-форм с несоизмеримыми периодами. Функц. анализ и его прил., 25, вып. 2, 1-12 (1991).

2. Арнольд В. И. Полиинтегрируемые потоки. Алгебра и анализ, 4, вып. 6, 54-62 (1992).

3. Дынников И. А. О пересечениях поверхностей уровня псевдопериодических функций. УМН, 49, вып. 1, 213-214 (1994).

Московский государственный

университет им. М. В. Ломоносова, механико-математический факультет
Поступило в редакцию 6 апреля 1994 г. 